\title{
An Approach Towards Image Edge Detection Based on Interval-Valued Fuzzy Mathematical Morphology and Admissible Orders
}

\author{
Peter Sussner $^{a}$ and Lisbeth Corbacho Carazas ${ }^{b}$ \\ ${ }^{a}$ Dept. of Applied Mathematics, IMECC, University of Campinas, SP, Brazil, sussner@ime.unicamp.br \\ ${ }^{b}$ Dept. of Applied Mathematics, IMECC, University of Campinas, SP, Brazil, ra162526@ime.unicamp.br
}

\begin{abstract}
Edge detection is an important step for preprocessing digital images before more advanced methods of image analysis such as segmentation can be applied. There are an infinite number of edge detectors that can be derived from pairs of fuzzy dilation and erosion operators. Usually, an edge detector is based on the incorrect assumption that there is no uncertainty regarding the pixel values of the given digital image. The approaches presented in this paper do not rely on this assumption. Instead, the uncertainty regarding the pixel values is modelled in terms of an interval-valued image. After an application of an interval-valued fuzzy dilation and erosion, we are able to produce a binary edge image after a number of steps including an order-preserving transformation based on an admissible order.
\end{abstract}

Keywords: Image edge detection, Intervalvalued fuzzy mathematical morphology, Morphological gradient, Admissible order.

\section{Introduction}

One of the problems one naturally faces when processing a digital image is the inherent uncertainty in the pixel values of a digital image due to image capture [13]. Even if the digital image is noiseless and if there is only one take of the image, uncertainty in the pixel values arises due to the following facts [11]

1. Quantization or Tonal Error: Any device will round captured values up or down so as to obtain one of the allowed values.

2. Discretization or Spatial Error: Since objects and surfaces are continuous in nature, their two- dimensional representation should be given by images whose point sets are continuous subsets of $\mathbb{R}^{2}$. Since a two-dimensional digital image has in practice a finite resolution, its point set can be assumed to be a rectangular subset $P$ of $\mathbb{Z}^{2}$. Due to the difficulties in mapping pixel values on an infinite grid to pixel values on a finite grid, the values on $P$ may be erroneously dislocated by one position in any direction.

This uncertainty can be modelled by converting a given digital image $O$ to an interval-valued image $A$ [11]. Since an interval-valued image can be viewed as an interval-valued fuzzy set, it can be processed using techiques of interval-valued fuzzy mathematical morphology (IV-FMM). For example, an interval-valued morphological gradient of a given interval-valued image can be computed in terms of a difference between the result of an interval-valued fuzzy (IV-fuzzy) erosion and an IV-fuzzy dilation [13, 15]. This difference between interval-valued fuzzy sets should be based on the difference between closed subintervals of $[0,1]$. The resulting interval-valued morphological gradient generalizes the fuzzy morphological gradient and can be mapped to a IV-fuzzy set or - in the language of type-2 fuzzy set theory - to a closed interval type- 2 fuzzy set.

Each pixel value of an interval-valued morphological gradient contains useful information regarding the strength of the edge and the uncertainty with respect to this strength. However, our goal in this paper is to produce binary edges that are one pixel wide [5]. A possible way to achieve this goal is to type reduce the corresponding interval type- 2 fuzzy set by using a convex combination of the upper and lower bounds so as to be able to apply techniques of thinning and binarization such as non-maximum suppression and hysteresis.

In this paper, we also adopt a completely different approach: We first apply an order-preserving transformation given by an admissible order [4] to the IV-fuzzy 
dilation and erosion images. As a result, we obtain regular fuzzy images, corresponding to a dilation and an erosion, whose difference can be computed as usual. This difference image can be interpreted as a grayscale morphological gradient image and allows for the application of conventional thinning and binarization techniques. We compare the quality of the binary edge images produced by using different admissible orders with the ones produced by using different convex combinations of the upper and lower bounds of an IV-fuzzy morphological gradient. Our comparison also includes the well-known Canny edge detector and a fuzzy morphological edge detector applied to the IV-fuzzy image $A$ after linearly ordering its pixel values.

The paper is organized as follows. Section 2 briefly reviews some pertinent lattice-theoretical concepts including admissible orders. Section 3 recalls some notions of $\mathbb{L}$-fuzzy set theory and $\mathbb{L}$-fuzzy mathematical morphology with an emphasis on IV-fuzzy set theory and IV-FMM. Section 4 presents our approach towards edge detection based on IV-FMM and admissible orders. Section 5 provides some experimental results.

\section{Some Concepts of Lattice Theory}

In this paper, we propose an edge detector based on IV-FMM and admissible orders, both of which draw on lattice and order theory. Let us review some basic concepts.

\subsection{Complete Lattices and Order-Preserving Mappings}

A partially ordered set or poset is a non-empty set together with a reflexive, antisymmetric and transitive binary relation " $\leqslant$ " [9]. If $(\mathbb{L}, \leqslant)$ is a poset and $x \leqslant y$ or $y \leqslant x$ for all $x, y \in \mathbb{L}$, then $\leqslant$ is called a total or linear order and $(\mathbb{L}, \leqslant)$ is called a totally ordered set or a chain. A partial order $\leqslant$ on $\mathbb{L} \neq \varnothing$ induces the notions of supremum and infimum. Let $X \subseteq \mathbb{L}$, where $(\mathbb{L}, \leqslant)$ is a poset. One refers to $u, l \in \mathbb{L}$ as an upper bound and a lower bound of $X$ if $x \leqslant u \forall x \in X$ and $l \leqslant x \forall x \in X$, respectively. If $u \in \mathbb{L}$ is the least upper bound of $X$, then $u$ is denoted $\bigvee X$ and called the supremum of $X$. If $l \in \mathbb{L}$ is the greatest lower bound of $X$, then $l$ is denoted $\bigwedge X$ and called the infimum of $X$. If $X=\{x, y\}$, then $\bigvee X$ and $\bigwedge X$ are respectively denoted $x \vee y$ and $x \wedge y$. If $X=\left\{x_{j} \mid j \in J\right\}$, then one writes $\bigvee_{j \in J} x_{j}$ and $\bigwedge_{j \in J} x_{j}$ instead of $\bigvee X$ and $\bigwedge X$, respectively.

A poset $(\mathbb{L}, \leqslant)$ such that $x \vee y$ and $x \wedge y$ exist in $\mathbb{L}$ for all $x, y \in \mathbb{L}$ is called a lattice. For simplicity, we write $\mathbb{L}$ instead of $(\mathbb{L}, \leqslant)$ if the partial order $\leqslant$ is evident from the context. Let us also clarify that the same symbol $\leqslant$ is often used to denote different partial orders. A lattice $\mathbb{L}$ is called complete, if for every $X \subseteq \mathbb{L}$ we have that $\bigvee X$ and $\bigwedge X$ exist in $\mathbb{L}$. In this case, the symbols $1_{\mathbb{L}}$ and $0_{\mathbb{L}}$ are used to denote $\bigvee \mathbb{L}$ and $\bigwedge \mathbb{L}$. If $\mathbb{L}$ is a lattice and $X \neq \varnothing$, then we have:

1. $\mathbb{L}^{n}=\mathbb{L} \times \ldots \times \mathbb{L}$ is a lattice with the following partial order:

$$
\begin{gathered}
\left(x_{1}, \ldots, x_{n}\right) \leqslant\left(y_{1}, \ldots, y_{n}\right) \Leftrightarrow x_{i} \leqslant y_{i}, \\
\forall i=1, \ldots, n .
\end{gathered}
$$

2. $\mathbb{L}^{m \times n}$ is a lattice with the following partial order:

$$
\begin{array}{r}
S \leqslant T \Leftrightarrow s_{i j} \leqslant t_{i j}, \\
\forall j=1, \ldots, m, \forall i=1, \ldots, n .
\end{array}
$$

3. $\mathbb{L}^{X}=\{f: X \rightarrow \mathbb{L}\}$ is a a lattice with the following partial order:

$$
f \leqslant g \Leftrightarrow f(x) \leqslant g(x) \forall x \in X
$$

4. The class of all graphs of functions in $\mathbb{L}^{X}$, denoted using the symbol $\mathcal{F}_{\mathbb{L}}(X)$, is lattice with the partial order given as follows. If $A=\left\{\left(x, \mu_{A}(x)\right) \mid x \in X\right\}$ and $B=\left\{\left(x, \mu_{A}(x)\right) \mid x \in X\right\}$, then

$$
A \leqslant B \Leftrightarrow \mu_{A} \leqslant \mu_{B}
$$

Recall that $\mu_{A} \in \mathbb{L}^{X}$ is referred to as the membership function of $A$ if $A=\left\{\left(x, \mu_{A}(x)\right) \mid x \in X\right\}$. An element of $\mathcal{F}_{\mathbb{L}}(X)$ is called an $\mathbb{L}$-fuzzy set. For simplicity, we write $A(x)$ instead of $\mu_{A}(x)$.

If the lattice $\mathbb{L}$ is complete, then the lattices $\mathbb{L}^{n}, \mathbb{L}^{m \times n}$, $\mathbb{L}^{X}$, and $\mathcal{F}_{\mathbb{L}}(X)$ are complete as well. Let us give some concrete examples of complete lattices:

1. The unit interval $[0,1]$ with the usual linear order;

2. The set of all closed, non-empty subintervals of $[0,1]$, denoted $\mathbb{I}$, with the partial ordering given by

$$
[\underline{x}, \bar{x}] \leqslant[\underline{y}, \bar{y}] \Leftrightarrow \underline{x} \leqslant \underline{y} \text { and } \bar{x} \leqslant \bar{y} .
$$

3. The power set of any $X \neq \varnothing$ with the partial ordering of (crisp) set inclusion;

4. The class of fuzzy sets on $X \neq \varnothing$, denoted $\mathcal{F}(X)$, with the partial ordering of inclusion of fuzzy sets. Note that $\mathcal{F}(X)=\mathcal{F}_{[0,1]}(X)$.

5. The class of IV-fuzzy sets on $X \neq \varnothing$, i.e., $\mathcal{F}_{\mathbb{I}}(X)$, with the partial order given by Equation 5 for the special case where $(\mathbb{L}, \leqslant)=(\mathbb{I}, \leqslant)$ (cf. Equation $6)$. 
A mapping $\phi: \mathbb{L} \rightarrow \mathbb{M}$, where $\left(\mathbb{L}, \leqslant_{\mathbb{L}}\right)$ and $\left(\mathbb{M}, \leqslant_{\mathbb{M}}\right)$ are lattices, establishes a relationship between these lattices. The mapping $\phi$ is said to be increasing or order-preserving if for all $x, y \in \mathbb{L}$ we have that $x \leqslant \mathbb{L}$ $y$ implies $\phi(x) \leqslant_{\mathbb{M}} \phi(y)$. Similarly, $\phi$ is said to be decreasing or order-reversing if for all $x, y \in \mathbb{L}$ we have that $x \leqslant_{\mathbb{L}} y$ implies $\phi(y) \leqslant_{\mathbb{M}} \phi(x)$.

Note that these notions not only depend on the values $\phi(x) \in \mathbb{M}$ but also on the partial order $\leqslant_{\mathbb{M}}$. Even if $\mathbb{L}=\mathbb{M}$ and the mapping $\phi$ is the identity, i.e., $\phi(x)=x$ for all $x \in \mathbb{L}, \phi$ may not be order-preserving. As an example, consider $(\mathbb{L}, \leqslant \mathbb{L})=([0,1], \leqslant)$ and $(\mathbb{M}, \leqslant \mathbb{M})=$ $([0,1], \geqslant)$.

Consider $(\mathbb{I}, \leqslant)$, where $\leqslant$ is as in Equation 6 , and $(\mathbb{I}, \leq)$, where $\leq$ is an arbitrary linear order. We say that the linear order $\leq$ refines the partial order $\leqslant$ if $[\underline{x}, \bar{x}] \leqslant[y, \bar{y}]$ implies that $[\underline{x}, \bar{x}] \leq[y, \bar{y}]$. In other words, $\leq$ refines $\leqslant$ if mapping $(\mathbb{I}, \leqslant)$ to $(\overline{\mathbb{I}}, \leq)$ by changing nothing but the order is order-preserving.

Definition 2.1. An order $\leq$ on II is said to be admissible if

1. $\leq$ is a linear order,

2. $\leq$ refines the partial order $\leqslant$ of Equation 6 .

The following are examples of admissible orders:

1. $[\underline{x}, \bar{x}] \leq_{l e x 1}[\underline{y}, \bar{y}]$ if only if $\underline{x}<\underline{y}$ or $\underline{x}=\underline{y}$ and $\bar{x} \leqslant$ $\bar{y}$ (lexicographic-1 order),

2. $[\underline{x}, \bar{x}] \preceq_{l e x 2}[y, \bar{y}]$ if only if $\bar{x}<\bar{y}$ or $\bar{x}=\bar{y}$ and $\underline{x} \leqslant$ $\underline{y}$ (lexicographic-2 order).

Other examples of admissible orders can be generated by means of Proposition 3.2 of [4] that we can rephrase as follows:

Proposition 2.1. Let $f, g:[0,1]^{2} \rightarrow[0,1]$ be continuous aggregation functions that satisfy the property below:

$$
\begin{array}{r}
f\left(x_{1}, x_{2}\right)=f\left(y_{1}, y_{2}\right) \text { and } g\left(x_{1}, x_{2}\right)=g\left(y_{1}, y_{2}\right) \\
\Rightarrow\left(x_{1}, x_{2}\right)=\left(y_{1}, y_{2}\right) .
\end{array}
$$

The functions $f$ and $g$ give rise to the following admissible order on $\mathbb{I}$ :

$$
\begin{aligned}
& {[\underline{x}, \bar{x}] \leq_{f, g}[\underline{y}, \bar{y}] \Leftrightarrow} \\
& (f(\underline{x}, \bar{x}), g(\underline{x}, \bar{x})) \leqslant_{l e x 1}(f(\underline{y}, \bar{y}), g(\underline{y}, \bar{y}))
\end{aligned}
$$

Recall that $f:[0,1]^{2} \rightarrow[0,1]$ is an aggregation function if and only if $f$ is order-preserving, $f(0,0)=0$, and $f(1,1)=1$. A particular type of continuous aggregation functions $[0,1]^{2} \rightarrow[0,1]$ is given by the following function $K_{\alpha}$ that is defined as follows for every $\alpha \in[0,1][3]:$

$K_{\alpha}\left(x_{1}, x_{2}\right)=x_{1}+\alpha\left(x_{2}-x_{1}\right), \forall\left(x_{1}, x_{2}\right) \in[0,1]^{2}$.

Note that $K \alpha$ and $K_{\beta}$, where $\alpha \neq \beta \in[0,1]$, satisfy Equation 7. For every pair $(\alpha, \beta) \in[0,1]^{2}$ such that $\alpha \neq \beta$, one can generate the following admissible order $\varsigma_{\alpha, \beta}$ :

$\left[x_{1}, x_{2}\right] \leq_{\alpha, \beta}\left[y_{1}, y_{2}\right] \Leftrightarrow$

$\left(K_{\alpha}\left(x_{1}, x_{2}\right), K_{\beta}\left(x_{1}, x_{2}\right)\right) \leq_{l e x 1}\left(K_{\alpha}\left(y_{1}, y_{2}\right), K_{\beta}\left(y_{1}, y_{2}\right)\right)$

Given an arbitrary $\alpha \in[0,1]$, all admissible orders $\leq_{\alpha, \beta}$ on II such that $\beta>\alpha$ and all admissible orders $\leq_{\alpha, \beta}$ on II such that $\beta<\alpha$ coincide [4]. Consequently, we obtain the following properties:

1. $\rfloor_{\alpha, \beta}$ equals $\leq_{\alpha, 1}$ for every $\beta>\alpha \in[0,1[$.

2. $\rfloor_{\alpha, \beta}$ equals $\leq_{\alpha, 0}$ for every $\left.\left.\beta<\alpha \in\right] 0,1\right]$.

For $\alpha \in\left[0,1\left[\right.\right.$, the symbol $\preceq_{\alpha+}$ denotes $\preceq_{\alpha, 1}$ and, for $\alpha \in] 0,1]$, the symbol $\leq_{\alpha-}$ denotes $\leq_{\alpha, 0}$.

If $\mathbb{L}$ is a lattice (complete lattice) then the product of $n$ copies of poset $\mathbb{L}$ denoted by $\mathbb{L}^{n}$ with the partial order $\leqslant$ given by

$$
\left(x_{1}, \ldots, x_{n}\right) \leqslant 2\left(y_{1}, \ldots, y_{n}\right) \Leftrightarrow x_{i} \leqslant y_{i} \forall i=1, \ldots, n .
$$

is a lattice (complete lattice).

The following types of order-preserving mappings are defined in mathematical morphology:

Definition 2.2. Let $\varepsilon, \delta: \mathbb{L} \rightarrow \mathbb{M}$, where $\mathbb{L}, \mathbb{M}$ are complete lattices.

1. The mapping $\varepsilon$ is called a(n algebraic) erosion if the following equation is satisfied for every index set $J$ and for every set $\left\{x_{j} \mid j \in J\right\} \subseteq \mathbb{L}$ :

$$
\varepsilon\left(\bigwedge_{j \in J} x_{j}\right)=\bigwedge_{j \in J} \varepsilon\left(x_{j}\right) .
$$

2. The mapping $\delta$ is a(n algebraic) dilation if the following equation is satisfied for every index set $J$ and for every set $\left\{x_{j} \mid j \in J\right\} \subseteq \mathbb{L}$ :

$$
\delta\left(\bigvee_{j \in J} x_{j}\right)=\bigvee_{j \in J} \delta\left(x_{j}\right) .
$$

Definition 2.3. Let $\delta: \mathbb{L} \rightarrow \mathbb{M}$ and $\varepsilon: \mathbb{M} \rightarrow \mathbb{L}$. One refers to the pair $(\varepsilon, \delta)$ as an adjunction or says that $\varepsilon$ and $\delta$ are adjoint if only if

$$
x \leqslant \varepsilon(y) \Leftrightarrow \delta(x) \leqslant y \forall x \in \mathbb{L}, y \in \mathbb{M} .
$$

If $\varepsilon$ and $\delta$ are adjoint, then $\varepsilon$ is an algebraic erosion and $\delta$ is an algebraic dilation. 
An order-preserving mapping $\phi$ from a lattice $\mathbb{L}$ to a lattice $\mathbb{M}$ is called an order-embedding if the following property is satisfied for all $x, y \in \mathbb{L}$ :

$$
x \leqslant y \Leftrightarrow \phi(x) \leqslant \phi(y) .
$$

Suppose that the lattices $\mathbb{L}$ and $\mathbb{M}$ are complete. If $\phi: \mathbb{L} \rightarrow \mathbb{M}$ is both a bijection and an order-emdedding, then $\phi$ is called a complete lattice isomorphism. If there is a complete lattice isomorphism from $\mathbb{L}$ to $\mathbb{M}$, then $\mathbb{L}$ and $\mathbb{M}$ are said to be isomorphic and one writes $\mathbb{L} \simeq \mathbb{M}$. Assuming that $\mathbb{L}$ is a complete lattice, we have

$$
\begin{aligned}
& \text { 1. } \mathcal{F}_{\mathbb{L}}(X) \simeq \mathbb{L}^{X} \text {, if } X \neq \varnothing ; \\
& \text { 2. } \mathcal{F}_{\mathbb{L}}(X) \simeq \mathbb{L}^{n} \text {, if } X=\{1, \ldots, n\} ; \\
& \text { 3. } \mathcal{F}_{\mathbb{L}}(X) \simeq \mathbb{L}^{m \times n} \text {, if } X=\{1, \ldots, m\} \times\{1, \ldots, n\} .
\end{aligned}
$$

\section{$2.2 \quad \mathbb{L}$-fuzzy Mathematical morphology}

Mathematical morphology (MM) was originally conceived as a theory for image processing and analysis using so called structuring elements. The two basic operators of mathematical morphology, from which a variety of image filters such as opening, closing, openclose filter, morphological gradient, watershed, hit-ormiss transform, etc., can be constructed are erosion and dilation. In Section 2.1, we recalled the definitions of erosion and dilation as algebraic operators that commute respectively with the infimum and the supremum operator.

For all practical purposes, we need to address the definitions of a (morphological) erosion and a (morphological) dilation of an image by a structuring element. A general framework for these operators is given by $\mathbb{L}$-fuzzy sets, where $\mathbb{L}$ is a complete lattice. Therefore, we will from now on assume that $\mathbb{L}$ stands for an arbitrary complete lattice.

The theory of $\mathbb{L}$-fuzzy MM [15] generalizes binary, gray-scale, fuzzy, IV-fuzzy, intuitionistic fuzzy, bipolar fuzzy MM, etc. $[1,13,16]$. In $\mathbb{L}$-fuzzy MM, one defines (morphological) dilations and erosions in terms of $\mathbb{L}$-fuzzy conjunctions and implications whose definitions are given below. If $\mathbb{L}=[0,1]$ or $\mathbb{L}=\mathbb{I}$, we obtain fuzzy conjunctions and implications or IV-fuzzy conjunctions and implications, respectively.

Definition 2.4. An increasing mapping $\mathcal{C}: \mathbb{L} \times \mathbb{L} \rightarrow$ $\mathbb{L}$ is called an $\mathbb{L}_{-}$-fuzzy conjunction if $\mathcal{C}\left(0_{\mathbb{L}}, 0_{\mathbb{L}}\right)=$ $\mathcal{C}\left(0_{\mathbb{L}}, 1_{\mathbb{L}}\right)=\mathcal{C}\left(1_{\mathbb{L}}, 0_{\mathbb{L}}\right)=0_{\mathbb{L}}$ and $\mathcal{C}\left(1_{\mathbb{L}}, 1_{\mathbb{L}}\right)=1_{\mathbb{L}}$. If $\mathcal{C}$ is additionally commutative, associative and satisfies $\mathcal{C}\left(1_{\mathbb{L}}, x\right)=x$, then $\mathcal{C}$ is called an $\mathbb{L}$-fuzzy triangular norm or t-norm.

A binary operator $\mathcal{I}: \mathbb{L} \times \mathbb{L} \rightarrow \mathbb{L}$ is called an $\mathbb{L}$ fuzzy implication if $\mathcal{I}(\cdot, z)$ is decreasing for every $z \in \mathbb{L}$,
$\mathcal{I}(z, \cdot)$ is increasing for every $z \in \mathbb{L}$ and if $\mathcal{I}\left(0_{\mathbb{L}}, 0_{\mathbb{L}}\right)=$ $\mathcal{I}\left(0_{\mathbb{L}}, 1_{\mathbb{L}}\right)=\mathcal{I}\left(1_{\mathbb{L}}, 1_{\mathbb{L}}\right)=1_{\mathbb{L}}$ and $\mathcal{I}\left(1_{\mathbb{L}}, 0_{\mathbb{L}}\right)=0_{\mathbb{L}}$. An $\mathbb{L}$-fuzzy implication $\mathcal{I}$ is called a border implicator if $\mathcal{I}\left(1_{\mathbb{L}}, x\right)=x$ for every $x \in \mathbb{L}$. Note that if $\mathcal{I}$ is the adjoint implication of an $\mathbb{L}$-fuzzy t-norm, then $\mathcal{I}$ is a border implicator.

An $\mathbb{L}$-fuzzy implication $\mathcal{I}$ and conjunction $\mathcal{C}$ on $\mathbb{L}$ are said to be adjoint if only if $\mathcal{I}(z, \cdot)$ and $\mathcal{C}(z, \cdot)$ are adjoint for every $z \in \mathbb{L}$.

For instance, the Fodor implication and the nilpotent minimum, denoted $I_{F D}$ and $T_{n M}$, respectively, are known to be adjoint. The Kleene-Dienes implication, denoted $I_{K D}$ below, obviously satisfies $I_{K D} \leqslant I_{F D}$.

$$
\begin{aligned}
& T_{n M}(x, y)=\left\{\begin{array}{lr}
0 & \text { if } x+y \leqslant 1 \\
x \wedge y & \text { otherwise. }
\end{array}\right. \\
& I_{F D}(x, y)= \begin{cases}1 & \text { if } x \leqslant y, \\
(1-x) \vee y & x>y\end{cases} \\
& I_{K D}(x, y)=(1-x) \vee y \text {. }
\end{aligned}
$$

We are now ready to define the concepts of (morphological) $\mathbb{L}$-fuzzy erosion and dilation of an image $A \in \mathcal{F}_{\mathbb{L}}(X)$ by a structuring element $S \in \mathcal{F}_{\mathbb{L}}(X)$.

Definition 2.5. Let $A, S \in \mathcal{F}_{\mathbb{L}}(X)$ and let $\mathcal{E}_{\mathcal{I}}, \mathcal{D}_{\mathcal{C}}$ : $\mathcal{F}_{\mathbb{L}}(X) \times \mathcal{F}_{\mathbb{L}}(X) \rightarrow \mathcal{F}_{\mathbb{L}}(X)$ be defined as follows:

$$
\begin{aligned}
& \mathcal{E}_{\mathcal{I}}(A, S)(x)=\bigwedge_{y \in X} \mathcal{I}(S(y-x), A(y)) \\
& \mathcal{D}_{\mathcal{C}}(A, S)(x)=\bigvee_{y \in X} \mathcal{C}(S(x-y), A(y))
\end{aligned}
$$

We refer to the operator $\mathcal{E}_{\mathcal{I}}$ as the (morphological) $\mathbb{L}_{\text {- }}$ fuzzy erosion of the image $A$ by the structuring element (SE) $S$ and to the operator $\mathcal{D}_{\mathcal{C}}$ as the (morphological) $\mathbb{L}$-fuzzy dilation of the image $A$ by the structuring element (SE) $S$. The operators $\mathcal{E}_{\mathcal{I}}$ and $\mathcal{D}_{\mathcal{C}}$ are said to be adjoint if and only if $\mathcal{E}_{\mathcal{I}}(\cdot, S)$ and $\mathcal{D}_{\mathcal{C}}(\cdot, S)$ are adjoint for all $S \in \mathcal{F}_{\mathbb{L}}(X)$.

Remark 1. Generally speaking, a particular approach towards $\mathbb{L}$-fuzzy $\mathrm{MM}$ is given by a pair consisting of an $\mathbb{L}$-fuzzy erosion $\mathcal{E}_{\mathcal{I}}$ and an $\mathbb{L}$-fuzzy dilation $\mathcal{D}_{\mathcal{C}}$. One usually employs a pair $\left(\mathcal{E}_{\mathcal{I}}, \mathcal{D}_{\mathcal{C}}\right)$ such that $\mathcal{I}$ and $\mathcal{C}$ are adjoint since in this case $\mathcal{E}_{\mathcal{I}}$ and $\mathcal{D}_{\mathcal{C}}$ have several useful algebraic properties.

Proposition 2.2. Let $A, S \in \mathcal{F}_{\mathbb{L}}(X)$, where $X$ is a subset of the Euclidean space $\mathbb{R}^{d}$ or the digital space $\mathbb{Z}^{d}$ that contains $\mathbf{0}=(0, \ldots, 0)^{T}$ If $S(\mathbf{0})=1_{\mathbb{L}}, \mathcal{C}$ is an $\mathbb{L}$-fuzzy t-norm, and $\mathcal{I}$ is an $\mathbb{L}$-fuzzy border implicator, then we have:

$$
\mathcal{E}_{\mathcal{I}}(A, S) \leqslant A \leqslant \mathcal{D}_{\mathcal{C}}(A, S)
$$


In IV-fuzzy MM, i.e., I-fuzzy MM, morphological erosions and dilations are respectively constructed using IV-fuzzy implications and conjunctions. There are several ways to construct IV-fuzzy logical operators from fuzzy logical operators. In this paper, we focus on the ones described below.

Definition 2.6. Let $C$ and $I$ be respectively be a fuzzy conjunction and a fuzzy implication. The pessimistic conjunction with representative $C$ and the optimistic implication $\mathcal{I}_{I}^{o}$ with representative $I$ are respectively denoted using the symbols $\mathcal{C}_{C}^{p}$ and $\mathcal{I}_{I}^{o}$. These IV-fuzzy logical operators are defined as follows for $\mathbf{x}=[\underline{x}, \bar{x}], \mathbf{y}=[\underline{y}, \bar{y}] \in \mathbb{I}:$

$$
\begin{array}{r}
\mathcal{C}_{C}^{p}(\mathbf{x}, \mathbf{y})=[C(\underline{x}, \underline{y}), C(\underline{x}, \bar{y}) \vee C(\bar{x}, \underline{y})], \\
\mathcal{I}_{I}^{o}(\mathbf{x}, \mathbf{y})=[I(\underline{x}, \underline{y}) \wedge I(\bar{x}, \bar{y}), I(\underline{x}, \bar{y})] .
\end{array}
$$

Let us recall the following fact which is a corollary of Theorem 13 of [15]:

Proposition 2.3. Let $C$ and $I$ be respectively be a fuzzy conjunction and a fuzzy implication. If $C$ satisfies $C(1, x)>0$ for all $x>0$ or if $I$ satisfies $I(1, x)<1$ for all $x<1$, then $\mathcal{I}_{I}^{o}$ and $\mathcal{C}_{C}^{p}$ are adjoint if only if $I$ and $C$ are adjoint.

\section{$3 \quad$ Three Approaches Towards Image Edge Detection Using IV-FMM}

As indicated in Section 1, we are confronted with the fact that the pixel values of digital images are inherently uncertain. This uncertainty can be taken into account by mapping a given image to an interval-valued image.

Suppose we are given an original gray-scale image $O$ on a point set $X$ such that $X \subseteq \mathbb{Z}^{d}$ with values in $\{0,1, \ldots, 255\}$. The image $O$ can be used to generate an interval-valued image $O_{I V}$ so as to count for the quantization and discretization errors [11]:

$$
O_{I V}(x)=\left[0 \vee \bigwedge_{y \in N(x)} O(y)-1,255 \wedge \bigvee_{y \in N(x)} O(x)+1\right]
$$

Here, $N(x)$ stands for a $3 \times 3$ neighborhood of $x \in X$. Subsequently, we mapped $O_{I V}$ to an IV-fuzzy image $A \in \mathcal{F}_{\mathbb{I}}(X)$ by normalizing $O_{I V}$. Note that the quantization and discretization errors are respectively modeled in terms of an interval of length 2 centered around the pixel value and the neighboring (assuming 8-connectivity) pixel values.

An edge image can be computed in terms of a morphological gradient. In this paper, the morphological gradient image is given by a difference between the results of the IV-fuzzy dilation and the IV-fuzzy erosion.

\subsection{Edge Detection Based on an Interval-Valued Morphological Gradient}

In this paper, we present two different approaches towards edge detection. The first one employs the difference between IV-fuzzy sets that is based on the following notion of difference between intervals [6]:

$$
\mathbf{x}-\mathbf{y}=[\underline{x}, \bar{x}]-[\underline{y}, \bar{y}]=[\underline{x}-\bar{y},(\underline{x}-\underline{y}) \vee(\bar{x}-\bar{y})] .
$$

Note that the interval $\mathbf{x}-\mathbf{y}$ may not be an element of $\mathbb{I}$ even if $\mathbf{x}, \mathbf{y} \in \mathbb{I}$ and $\mathbf{y} \leqslant \mathbf{x}$. Nevertheless, an intervalvalued morphological gradient image can be computed as the difference, given by Equation 25, between the IV-fuzzy sets $\mathcal{D}_{\mathcal{C}}(A, S)$ and $\mathcal{E}_{\mathcal{I}}(A, S)$. We use the symbol $\mathcal{D}_{\mathcal{C}}(A, S) \backslash \mathcal{E}_{\mathcal{I}}(A, S)$ to denote this interval-valued difference. Figure 1 illustrates this procedure in terms of an example.
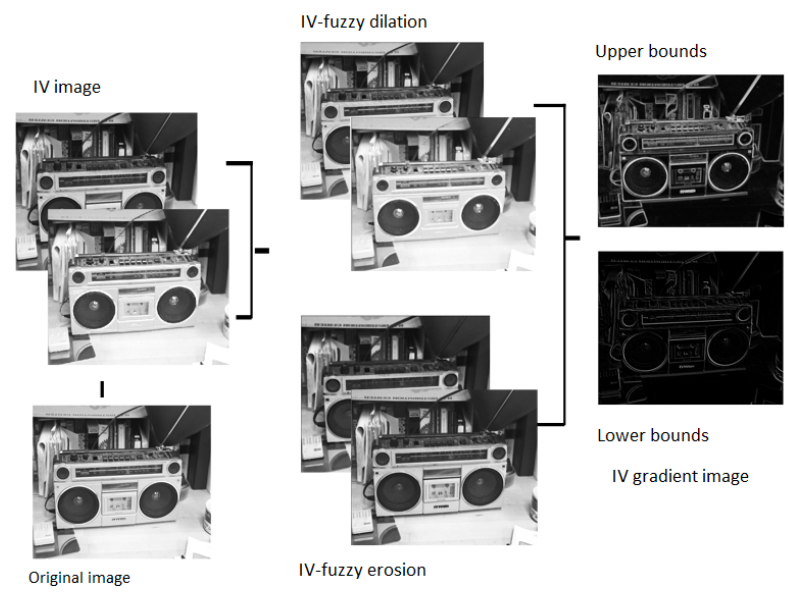

Figure 1: Sequence that leads to an interval-valued edge image.

Then we transformed the interval-valued morphological gradient image $\mathcal{D}_{\mathcal{C}}(A, S) \backslash \mathcal{E}_{\mathcal{I}}(A, S)$ into a grayscale morphological gradient that permits applying the usual thinning and binarization techniques in order to obtain a binary edge image that satisfies Canny's condition of one pixel width [5]. To this end, we computed a convex combination of the image $U$ corresponding to the upper bound and the image $L$ corresponding to the lower bound of $\mathcal{D}_{\mathcal{C}}(A, S) \backslash \mathcal{E}_{\mathcal{I}}(A, S)$.

\subsection{Edge Detection Using IV-FMM and Admissible Orders}

First, let us make the following observation: Although $\mathbb{I}$ is infinite, the values that the IV-fuzzy image $A \in$ $\mathcal{F}_{\mathbb{I}}(X)$ can assume are finite. Since the original image $O$ has values in $\{0, \ldots, 255\}$, there are exactly $\left(\begin{array}{c}256 \\ 2\end{array}\right)+$ $256=32896$ possible pixel values of $A$ in $\mathbb{I}$. These values are elements of $\mathbb{I}$ which can be linearly ordered 
using an admissible order. This procedure gives rise to a value set $\mathbb{L}=\{0,1, \ldots, 32895\}$ and to an image $A_{\mathbb{L}} \in \mathbb{L}^{X}$.

Consider an $\mathbb{L}$-fuzzy conjunction $C$, an $\mathbb{L}$-fuzzy implication $I$ (note that $C$ and $I$ are discrete fuzzy connectives [7]), and an $\mathrm{SE} S_{\mathbb{L}} \in \mathbb{L}^{X}$. If $C, I$ and $S_{\mathbb{L}}$ satisfy the conditions of Proposition 2.2, then a morphological gradient image with values in $\mathbb{L}$ is given by $\mathcal{D}_{C}\left(A_{\mathbb{L}}, S_{\mathbb{L}}\right)-\mathcal{E}_{I}\left(A_{\mathbb{L}}, S_{\mathbb{L}}\right)$.

A third approach is the following: First, one computes the images $D=\mathcal{D}_{\mathcal{C}}(A, S)$ and $E=\mathcal{E}_{\mathcal{I}}(A, S)$ using an IV-fuzzy conjunction $\mathcal{C}$, an IV-fuzzy implication $\mathcal{I}$, and an $\mathrm{SE} S \in \mathbb{I}^{X}$ such that $\mathcal{C}, \mathcal{I}$ and $S$ are as in Proposition 2.2. Then one uses an admissible order to map the IV-fuzzy images $D$ and $E$ to images $D_{\mathbb{L}}$ and $E_{\mathbb{L}}$ in $\mathbb{L}^{X}$, respectively. Note that this mapping is order-preserving. Thus, $E_{\mathbb{L}} \leqslant A_{\mathbb{L}} \leqslant D_{\mathbb{L}}$ which implies that a gray-scale morphological gradient $G_{\mathbb{L}} \in \mathbb{L}^{X}$ is given by the usual difference $D_{\mathbb{L}}-\mathcal{E}_{\mathbb{L}}$. Then $G_{\mathbb{L}}$ can be subjected to thinning and binarization in order to generate a binary edge image. Figure 2 illustrates the most important steps performed in this approach.

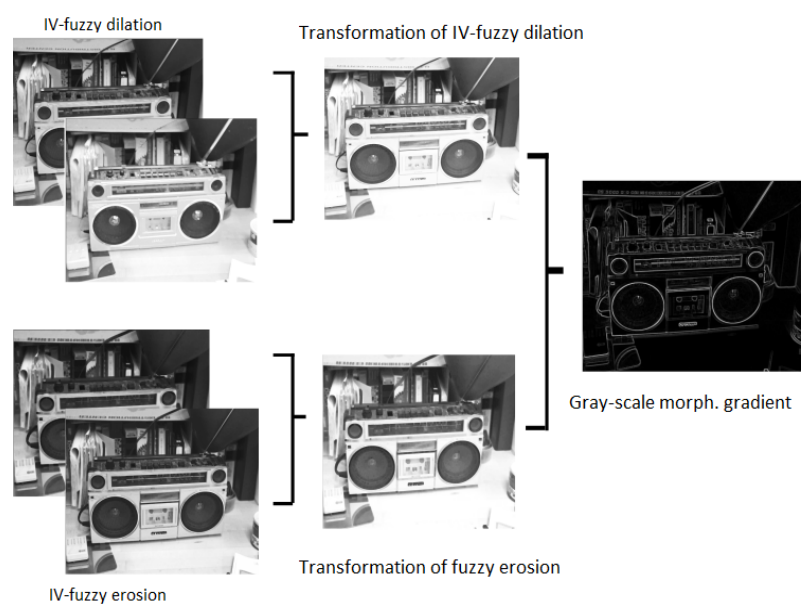

Figure 2: The figure shows from left to right: Results of the IV-fuzzy erosion and dilation, images obtained after linearly ordering interval-valued pixel values using an admissible order, gray-scale morphological gradient image computed from the previous two images.

\section{Some Experimental Results}

In our simulations, we considered the first 25 images of the public image dataset of the University of South Florida, including their respective ground truth images $[2]^{1}$. To begin with, we generated 25 IV-fuzzy images

\footnotetext{
${ }^{1}$ The images dataset can be downloaded from ftp://figment.csee.usf.edu/pub/ROC/
}

using these gray-scale images following the methodology of Lopez-Molina [11]. As to the choice of the structuring element and the fuzzy conjunction and implication that are the representatives of the IV-fuzzy conjunction and implication used in the IV-fuzzy dilation and erosion, a previous article of González-Hidalgo et al. on fuzzy morphological edge detectors [8] served as an inspiration. This article reveals that the pair $\left(T_{n M}, I_{K D}\right)$ is one of the two best configurations in terms of several statistical measures among 40 configuration for detecting the edges of 50 images, comprising the folder "Object" of this dataset. Also note that both of these fuzzy logical connectives are discrete, that $\mathcal{C}_{T_{n M}}^{p}$ is an IV-fuzzy t-norm and that $\mathcal{I}_{I_{K D}}^{o}$ is an IV-fuzzy border implicator. Therefore, the pair $\left(T_{n M}, I_{K D}\right)$ lends itself perfectly to applying the approaches described in Sections 3.1 and 3.2. We furthermore adopted the same structuring element used in Gonzalez-Hidalgo's work, that is

$$
S=\left(\begin{array}{ccc}
0.86 & 0.86 & 0.86 \\
0.86 & 1 & 0.86 \\
0.86 & 0.86 & 0.86
\end{array}\right)
$$

as well as the same methods for thinning the edges and binarizing the result $[7,8]$. Specifically, these methods are non-maximum supression (NMS) for thinning the edges [5] (using Kovesi's MATLAB code [10]), followed by hysteresis to binarize the image [5] with threshold values determined using the unsupervised approach by Medina-Carciner et al. [12]. Subsequently we compared the final result $(D E)$ (detected edges) with the ground truth $(G T)$ image. The latter is a binary edge image whose edges are one pixel wide. To this end, we used Pratt's figure of merit (FoM) that which is defined as follows [14]:

$$
\mathrm{FoM}=\frac{1}{\max \{|(D E)|(G T) \mid\}} \sum_{x \in D E} \frac{1}{1+a d^{2}} .
$$

Here $a \in \mathbb{R}^{+}$is a constant, $d$ is the distance between the detected edge and the ground truth edge. The symbols $|(D E)|$ and $|(G T)|$ denote respectively the numbers of detected and ground truth edge points. We used $a=1$ and the Euclidean distance.

Recall that our first approach depends on the parameter $\alpha$ in the convex combination $\alpha U+(1-\alpha) L$, where $U$ is the upper bound image and $L$ is the lower bound image shown in Figure 1. The effect on the gray-scale morphological gradient image when varying the parameter $\alpha$ is shown in Figure 3 .

In a similar vein, our second approach depends on the choice of $\leq_{\alpha+}$ or $\leq_{\alpha-}$. Figure 4 depicts the gray-scale morphological gradients for different choices of admissible orders. Note that $\leq_{0+}$ corresponds to

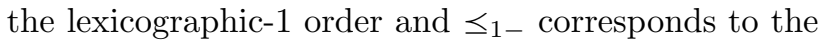
lexicographic-2 order. 


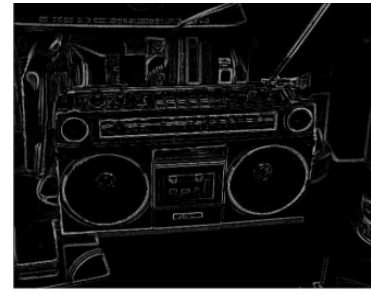

(a)

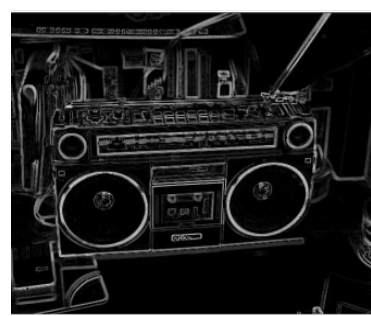

(c)

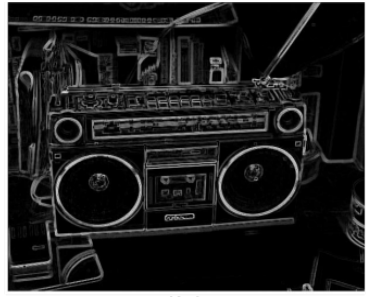

(b)

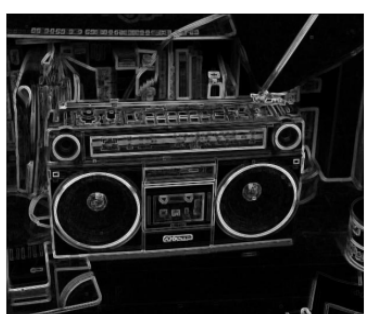

(d)
Figure 3: Gray-scale edges obtained by varying $\alpha$ that determines the convex combination of the upper and lower bound images of an IV morphological gradient (cf. 3.1): (a) $\alpha=0.25$ (b) $\alpha=0.5$ (c) $\alpha=0.45$ (d) $\alpha=1$.

We evaluated the performance of each edge detector in terms of the average of the FoM values over the 25 images under consideration. In the case of the intervalvalued morphological gradient method, we generated 51 edge detectors by considering $\alpha \in\{0,0.02, \ldots, 1\}$ as parameters in the convex combinations of the upper and lower bounds of the interval-valued morphological gradients. In a similar vein, we applied 51 edge detectors following the approach described in Section 3.2 by varying $\alpha \in\{0,0.02, \ldots, 1\}$ in the admissible orders of type $\leq_{\alpha-}$. Finally, we applied $K_{\alpha-}$ to $A \in \mathcal{F}_{\mathbb{I}}(X)$, obtained by normalizing $O_{I V}$ of Equation 24, and generated a binary edge image from the fuzzy morphological gradient image of $K_{\alpha-}(A)$. Figure 5 and Table 1 summarize the performances in terms of FoM values produced by the methods under consideration. Since the FoM values are similar for the methods based on $\varsigma_{\alpha-}$ and $\varsigma_{\alpha+}$, we omitted the latter.

Note that the approaches that involve ordering the interval values of the IV-fuzzy erosion and dilation images using an admissible order before subtracting one from the other outperform the approach that relies on computing a convex combination of the upper and lower bounds of the interval-valued morphological gradient. The latter reveals an approximately increasing performance with respect to $\alpha$. Note that the gray-scale values of the resulting morphological gradient image increase as $\alpha$ tends to 1 , leading to the detection of a larger number of edges.

Despite the fact, our IV-MM and admissible order based edge detection method was applied to an im-

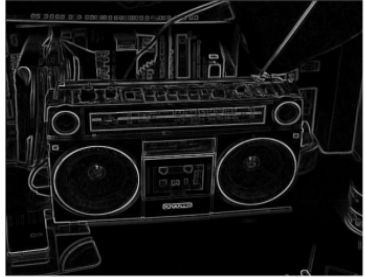

(a)

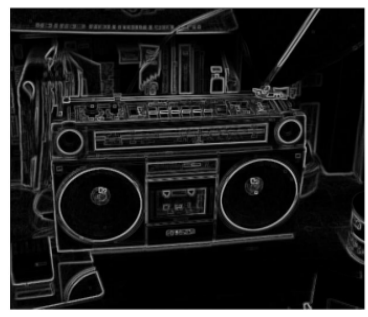

(c)

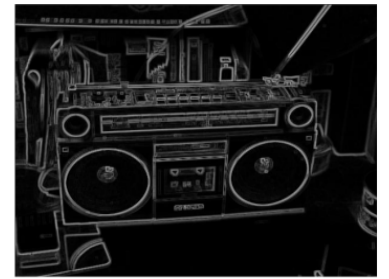

(b)

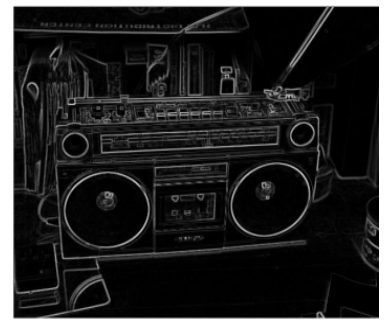

(d)
Figure 4: Gray-scale morphological gradients obtained by varying the admissible order in the approach of Section 3.2: (a) $\leq_{0.0008-}$ (b) $\preceq_{0.4-}$ (c) $\preceq_{0.78-}$ (d) $\preceq_{1-}$.

\begin{tabular}{l|l}
\hline Edge detectors & FoM \\
\hline \hline$K_{\alpha-}$ (IVF dilation) - $K_{\alpha-}$ (IVF erosion) & 0.34148 \\
Fuzzy morph. grad. of $K_{\alpha-}$ (IVF image) & 0.32506 \\
Convex combination IVF gradient & 0.32668 \\
Canny & 0.27015 \\
\hline
\end{tabular}

Table 1: Average of the FoM values over 25 images for the Canny method applied to the original image in comparison with the highest average FoMs achieved by the three methods for some $\alpha \in\{0,0.02, \ldots, 1\}$.

age with uncertain pixel values, this method produced better FoM values for most $\leq_{\alpha-}$ and $\leq_{\alpha+}$ orders than the popular Canny edge detector applied to the original image without uncertainty.

\section{Concluding Remarks}

In this paper, we proposed a new edge detection method for IV-fuzzy images. To this end, we applied an admissible order to the IV-fuzzy erosion and dilation images and subtracted the former from the latter. In our experiments, we binarized the resulting morphological gradient and computed the FoM. Our approach produced higher FoM values than the grayscale morphological gradients of $K_{\alpha-}(A), K_{\alpha+}(A)$ and than forming the convex combination of the upper and lower bounds of $\mathcal{D}_{\mathcal{C}}(A, S) \backslash \mathcal{E}_{\mathcal{I}}(A, S)$. Although we used an IV-fuzzy image $A$ that contains less information than the original image $O$, our approach outperformed the classical Canny method.

\section{Acknowledgement}




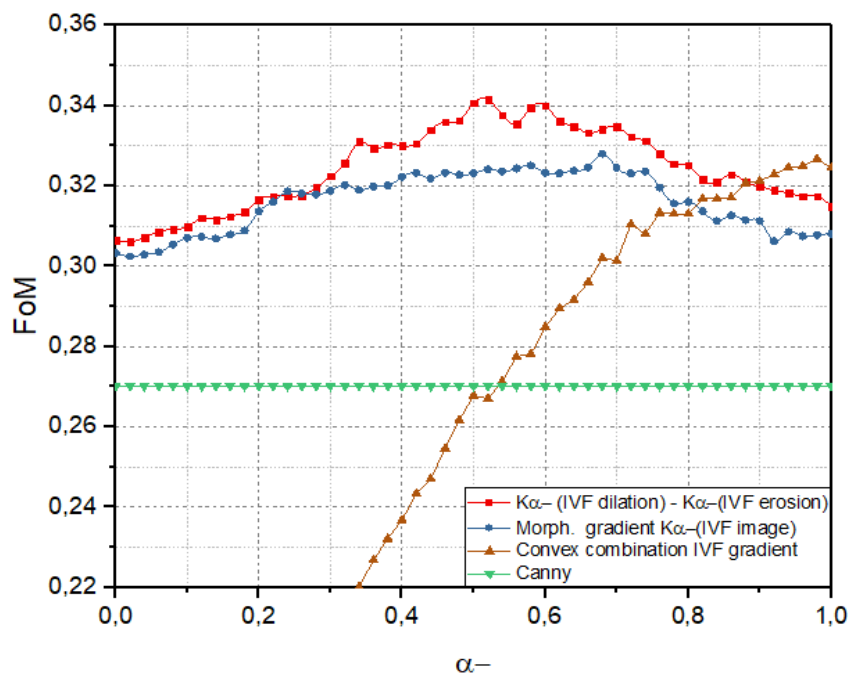

Figure 5: Average FoM over 25 images for the Canny method applied to the original image in comparison with the average FoMs achieved by three different methods that were applied to IV-fuzzy images $A \in \mathcal{F}_{\mathbb{I}}(X)$ for $\alpha \in\{0,0.02, \ldots, 1\}$.

This work was supported in part by CAPES, CNPq under grant no. 313145/2017-2 and FAPESP under grant no. 2018/13657-1.

\section{References}

[1] I. Bloch, Mathematical morphology on bipolar fuzzy sets: general algebraic framework, International Journal of Approximate Reasoning 53 (7) (2012) 1031-1060.

[2] K. Bowyer, C. Kranenburg, S. Dougherty, Edge detector evaluation using empirical ROC curves, Computer Vision and Image Understanding 84 (1) (2001) 77-103.

[3] H. Bustince, T. Calvo, B. De Baets, J. Fodor, R. Mesiar, J. Montero, D. Paternain, A. Pradera, A class of aggregation functions encompassing two-dimensional OWA operators, Information Sciences 180 (10) (2010) 1977-1989.

[4] H. Bustince, J. Fernández, A. Kolesárová, R. Mesiar, Generation of linear orders for intervals by means of aggregation functions, Fuzzy Sets and Systems 220 (2013) 69-77.

[5] J. F. Canny, A computational approach to edgedetection, IEEE Transactions on Pattern Analysis and Machine Intelligence 8 (1986) 679-700.

[6] G. Deschrijver, C. Cornelis, Representability in interval-valued fuzzy set theory, International
Journal of Uncertainty, Fuzziness and KnowledgeBased Systems 15 (03) (2007) 345-361.

[7] M. González-Hidalgo, S. Massanet, A fuzzy mathematical morphology based on discrete t-norms: fundamentals and applications to image processing, Soft Computing 18 (11) (2014) 2297-2311.

[8] M. González-Hidalgo, S. Massanet, A. Mir, D. Ruiz-Aguilera, On the choice of the pair conjunction-implication into the fuzzy morphological edge detector, IEEE Transactions on Fuzzy Systems 23 (4) (2015) 872-884.

[9] G. A. Grätzer, Lattice Theory: First Concepts and Distributive Lattices, W. H. Freeman, San Francisco, CA, 1971.

[10] P. D. Kovesi, Matlab and Octave functions for computer vision and image processing, Centre for Exploration Targeting, School of Earth and Environment, The University of Western Australia, available from: http://www. csse. uwa. edu. au/ pk/research/matlabfns 147 (2000) 230.

[11] C. Lopez-Molina, C. Marco-Detchart, J. Cerron, H. Bustince, B. De Baets, Gradient extraction operators for discrete interval-valued data, in: 16th IFSA World Congress; 9th Conference of the European Society for Fuzzy Logic and Technology, Vol. 89, Atlantis Press, 2015, pp. 836-843.

[12] R. Medina-Carnicer, R. Munoz-Salinas, E. Yeguas-Bolivar, L. Diaz-Mas, A novel method to look for the hysteresis thresholds for the Canny edge detector, Pattern Recognition 44 (6) (2011) 1201-1211.

[13] M. Nachtegael, P. Sussner, T. Mélange, E. E. Kerre, On the role of complete lattices in mathematical morphology: From tool to uncertainty model, Information Sciences 181 (10) (2011) 1971-1988.

[14] W. K. Pratt, Digital image processing: PIKS Scientific inside, Vol. 4, Wiley-interscience Hoboken, New Jersey, 2007.

[15] P. Sussner, M. Nachtegael, T. Mélange, G. Deschrijver, E. Esmi, E. Kerre, Interval-valued and intuitionistic fuzzy mathematical morphologies as special cases of $\mathbb{L}$-fuzzy mathematical morphology, Journal of Mathematical Imaging and Vision 43 (1) (2012) 50-71.

[16] P. Sussner, M. E. Valle, Classification of fuzzy mathematical morphologies based on concepts of inclusion measure and duality, Journal of Mathematical Imaging and Vision 32 (2) (2008) 139159. 